\title{
The Analysis of the Muscle Activity in Individual Work Sections of the Sowing Process
}

\author{
Sang Mi Lee*, Gyung Mee Gim, Seon Hee Jeong, Sun Jin Jeong, Yoonah Jang, Kyung Sook Han, and Hyun Jin Jang \\ Agricultural Research Division, National Institute of Horticultural and Herbal Science, Rural Development Administration, Jeonju 55365, \\ Korea
}

\begin{abstract}
This study was conducted to analyze the muscle activity in the right forearm and shoulderwhile doing the sowing activity by muscle type and work section, and to find the possibility of the utilization of sowing as a muscle exercise and rehabilitative activity based on the results of the analysis. The results showed that the activity of 7 muscles including the upper trapezius near the neck, the upper trapezius near the shoulder, the middle deltoid, the biceps brachii, the triceps brachii, the brachioradialis, and the flexor carpi ulnaris were significantly higher than the stable state. In addition, the muscle activity in the sections of mixing the soil, filling the tray with the soil, sowing, writing the label, and watering was significantly higher than the stable state. In particular, the muscle activity of the biceps brachii and the triceps brachii was statistically significantly high in the section of filling the tray with the soil, which indicates that the task of filling the tray with the soil can be utilized as an exercise to activate the biceps brachii and the triceps brachii. In addition, the muscle activity of the brachioradialis was significantly high in all the work sections, which indicates that the sowing process can be utilized to exercise the brachioradialis in particular. These results can be used as the basic data about the activated muscle types and degrees throughout the sowing process. These results can be also used to induce the activation of specific muscles, and the overall exercise and rehabilitation of the forearms.
\end{abstract}

Keywords: muscle exercise and rehabilitative utilization, right forearm and shoulder muscles

\section{Introduction}

Horticultural activities involve sensory participation and active participation which are key factors for the therapeutic utilization of horticultural activities (Matsuo, 1998). Some earlier studies on sensory participation using plants analyzed the impact of visual stimuli, using foliage plants or flower, or olfactory stimuli, using the scent of herbs or flowers, on humans (Jang et al., 2016; Lee et al., 2012). Some studies on active participation focused on the ROM (range of motion) and muscle activity of flower arrangement (Lee et al., 2012), and the exercise intensity of activities such as making a vegetable garden and maintaining a garden (Park et al., 2015). In addition, the effect of a therapeutic horticultural program or a horticultural activity program in which multiple sessions are conducted in series has been widely researched in the social horticultural field. Still, it is necessary to analyze the effect of individual activities that comprise such programs in 
order to identify the effect of horticultural therapy and therapeutic horticulture on humans (Relf, 2006), and the results of analysis can be actively used to select specific horticultural activities suitable for participants in horticultural therapy. However, there are many different types of horticultural activities, and, thus, it would be better to conduct such studies not in an intermittent and partial manner, but they need to be planned and conducted in stages in a continuous manner. In the process of planning a phased and continuous study on the effect of individual horticultural activities, sowing, the beginning of the life of plants, was selected as the first activity in this study, since it is most frequently used as a general activity in therapeutic horticultural programs (Lim, 2016). Reactions to the selected activity can be assessed from different perspectives, such as physical, cognitive, psychological and social aspects. This study focused on, among physical reactions, the activity of the forearm and shoulder muscles mobilized in the process of sowing, analyzed the muscle activity by the work section of sowing and the type of muscles, and examined the possibility of the utilization of sowing as a muscle exercise and a rehabilitative activity.

\section{Methods}

\section{Subjects}

This study was conducted among 37 right-handed females in their 20s and 60s who had an experience of sowing. Data that had errors were excluded, and, thus, the data on 30 participants were used in the final analysis. Those who voluntarily consented to participate in this study were informed of the purpose and process of this study, and signed the consent form. The copies of the consent form were kept by each participant and the researcher. The average age of subjects was $38.3 \pm 9.18$ years; the average height, $161 \pm 7.26 \mathrm{~cm}$; and the average weight, $56.6 \pm 4.38 \mathrm{~kg}$.

\section{Preparation of laboratory}

This study was conducted in a laboratory (width: $250 \mathrm{~cm}$, length: $320 \mathrm{~cm}$, height: $265 \mathrm{~cm}$ ) in the National Institute of Horticultural and Herbal Science. A white-color table (width: $100 \mathrm{~cm}$, length: $50 \mathrm{~cm}$, height: $60 \mathrm{~cm}$ ) and a chair with the back were placed in the room, and a tripod and a camera used to record the movement of subjects were placed $1 \mathrm{~m}$ away from the right side of subjects. Process analysis paper was attached $150 \mathrm{~cm}$ away from the front of subjects for them to refer to the process of sowing. The temperature of the laboratory was $25 \sim 26^{\circ} \mathrm{C}$; humidity, $60-70 \%$; the level of noise, 61-64 db; and the level of illumination, approximately 500 lux.

\section{Worktable setting}

A stainless bowl (width: $35 \mathrm{~cm}$, height: $12 \mathrm{~cm}$ ) was placed in the right bottom corner of the worktable, and a pair of ivory-color scissors (length: $20 \mathrm{~cm}$, weight: $63 \mathrm{~g}$ ), to the right of the bowl. A watering can filled with $300 \mathrm{~mL}$ of water (width: $38 \mathrm{~cm}$, height: $15 \mathrm{~cm}$, total weight: $465 \mathrm{~g}$ ) was placed in front of the pair of scissors, and a zipper bag filled with 4 L of horticultural bed soil (width: $27.5 \mathrm{~cm}$, length: $29 \mathrm{~cm}$, total weight: $525 \mathrm{~g}$ ), to the left of the watering can. A beaker filled with $400 \mathrm{~mL}$ of water (width: $12 \mathrm{~cm}$, height: $11 \mathrm{~cm}$, total weight $526 \mathrm{~g}$ ) was placed to the left of the zipperbag, and a black plastic trowel (length: $32 \mathrm{~cm}$, width: $10 \mathrm{~cm}$, weight: $100 \mathrm{~g}$ ), to the left of the beaker. A 50-cell black empty plug tray (width: $54 \mathrm{~cm}$, length: $28 \mathrm{~cm}$, height: $5 \mathrm{~cm}$, total weight: $125 \mathrm{~g}$ ) was placed to the left of the trowel, and a white label (width: $50 \mathrm{~mm}$, length: $97 \mathrm{~mm}$, weight: $2 \mathrm{~g}$ ), to the left of the plug tray. A black pen (length: $13.5 \mathrm{~cm}$, weight: $8 \mathrm{~g}$ ) was placed to the left of the label, and a transparent plastic petri dish (diameter: $9 \mathrm{~cm}$, height: $1.5 \mathrm{~cm}$, total weight: $22 \mathrm{~g}$ ) with lettuce seeds, to the left of the pen (Fig. 1). 


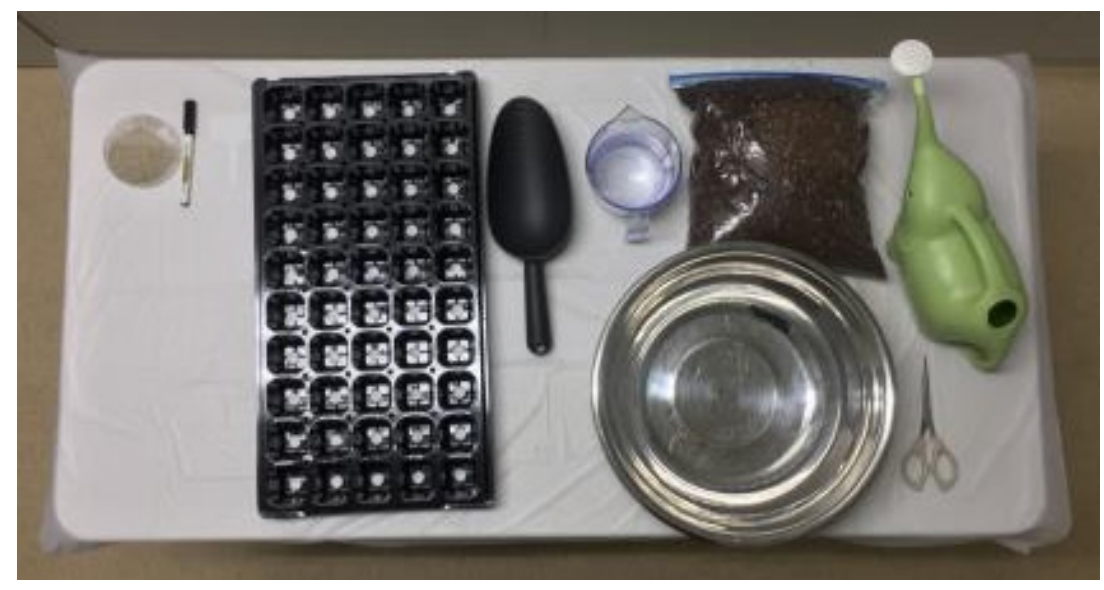

Figure 1. Table setting for the experiment.

\section{Instructions for subjects before the experiment}

Subjects were instructed to have a deep sleep and not to drink the day before the experiment, and not to exercise excessively before the experiment. They were also instructed not to drink coffee or take a stimulant (energy drinks) 1 hour before the experiment, which is because drinking, coffee or stimulants can affect brain waves. Subjects were instructed to wash and dry their hair right before coming to the experiment in order to collect electrical signals transferred through the scalp more efficiently by removing dirt from the scalp. They were well-informed of the procedure of sowing. On the day of the experiment, subjects took a rest for over 10 minutes after arriving at the laboratory. Researchers explained the purpose and process of this study to subjects again, and subjects were ensured to read and sign the consent form, and to change into clothes for the experiment. Electrodes were attached to the several parts of the body in the laboratory.

\section{Attaching electrodes and measuring muscle activities}

EMG (electromyography) was adapted to measure and analyze the muscle activity of subjects. Electrodes were attached to the skin, and electrical changes in the muscles were observed. EMG is a useful method to predict the strength of the muscles through electrical signals generated by muscular contraction. The higher the EMG level, the higher the strength of the muscles, and the higher the muscle activity (Go et al., 1997). To prevent any obstruction to the activities of this study, 8-channel wireless surface electromyography (sEMG) and BIOS-mini (Biobrain Inc., Daejeon, Korea), an ECG (electrocardiogram) meter, were used. Since the measured EMG signals are analogue, they were discretely digitized into $1,000 \mathrm{~Hz}$ sampling frequency to produce indicators in a computing system, and the digital resolution was 24 bit. The converted digital signals were wirelessly transferred to a personal computer. RMS (Root Mean Square, unit: $\mu \mathrm{V}$ ) indicators that reflect the muscle activity were calculated using BioScan (Biobrain inc., Daejeon, Korea), an EMG analysis program, and $4 \sim 500 \mathrm{~Hz}$ band-pass filters were applied to the program. To remove the noise of the power supply, 60, 120 and $180 \mathrm{~Hz}$ Nocth filters were applied. Table 1 shows the photos and specifications of devices used in this study.

To measure EMG, a total of 7 muscles were selected as follows: the upper trapezius near the neck in the right forearm; the upper trapezius near the shoulder; the middle deltoid; the biceps brachii; the triceps brachii; the brachioradialis; and the flexor carpi ulnaris. The areas where electrodes were attached were referred to the method of Netter (2000). The selected muscles are as shown in Fig. 2. To quantify the baseline of the activities of the muscles, the EMG of subjects was measured in a stable state in a seated position for 1 minute before and after the activity respectively. 
Table 1. Specifications of the EMG measuring device used in the experiment.

\begin{tabular}{lc}
\hline \multicolumn{1}{c}{ Measuring device } & Specifications \\
\hline Biosignal channel & up to 8 channels \\
Sampling Rate & $1 \mathrm{kHz} /$ channel \\
Noise & $<8 \mathrm{uVpp}$ \\
AD Conversion & 24 bit [Analog to Digital] \\
Input Impedance & $>1$ Gohm \\
Communication Interface & Wifi \\
\hline
\end{tabular}

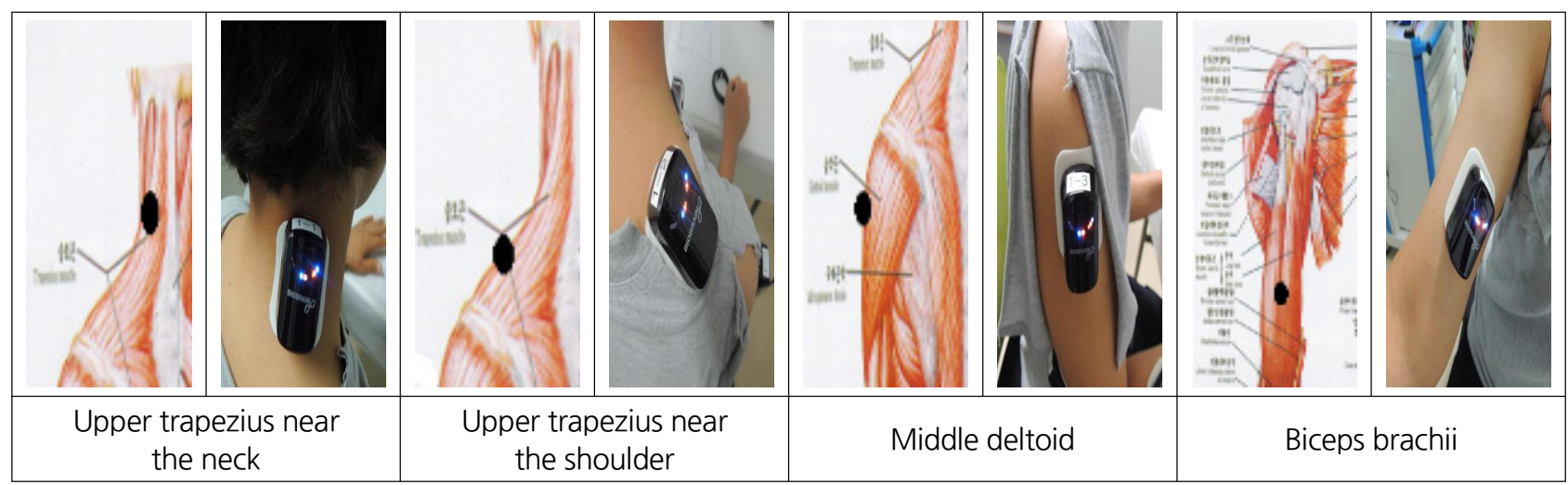

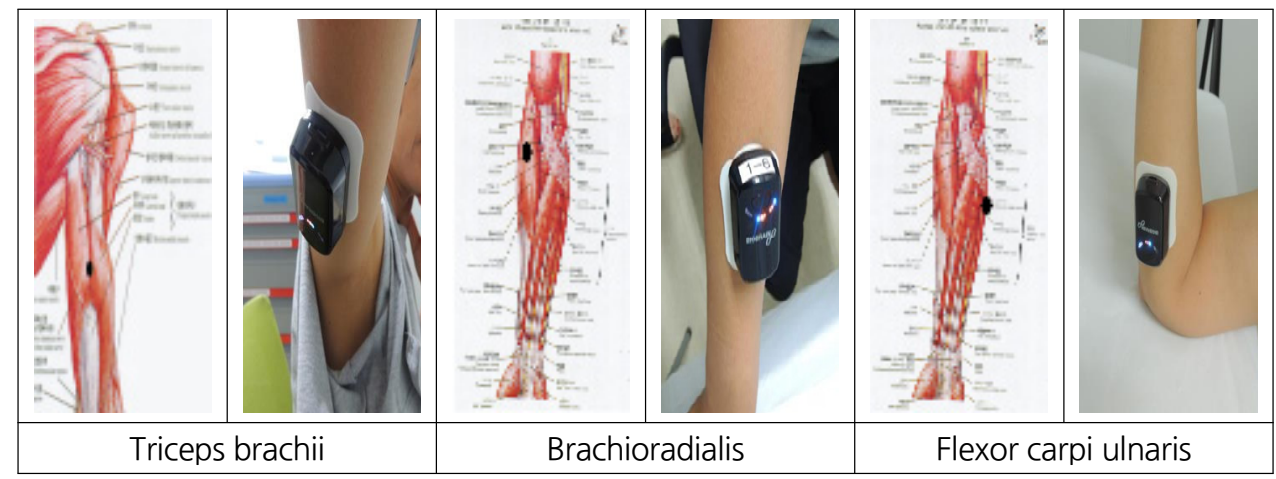

Figure 2. Forearm muscles where EMG electrodes were attached.

\section{Detailed tasks of the sowing process}

The sowing process was divided into 5 sections as follows: mixing the soil; filling the tray with the soil; sowing; writing the label; and watering. The $1^{\text {st }}$ section was composed of 6 tasks; the $2^{\text {nd }}$ section, 5 tasks; the $3^{\text {rd }}$ section, 5 tasks; the $4^{\text {th }}$ section, 2 tasks; and the $5^{\text {th }}$ section, 3 tasks, and they are listed in Table 2 . Subjects were instructed to perform the tasks by referring to the process analysis paper attached to the wall in front of the subjects. This experiment was conducted from September to November, 2016.

\section{Data processing and statistical analysis}

To analyze the activity of the muscles, the measured data were statistically analyzed as follows. Descriptive statistics were used to obtain the average and standard deviation values of the muscle activity by work section, work time and muscle type. One-way ANOVA and post-hoc analysis were conducted using SPSS Win Ver. 22.0, and Duncan's method 
Table 2. Work sections and tasks of the sowing process.

\begin{tabular}{|c|c|}
\hline Work section & Task \\
\hline \multirow{6}{*}{ 1. Mixing the soil } & 1) Bring the bowl to the center of the body. \\
\hline & 2) Cut the bag containing the soil with scissors. \\
\hline & 3) Pour all the soil into the bowl. \\
\hline & 4) Pour all the water in a plastic beaker into the bowl. \\
\hline & 5) Mix the water and soil evenly with your hand. \\
\hline & 6) Push the bowl slightly to the right. \\
\hline \multirow{5}{*}{ 2. Filing the tray with the soil } & 7) Bring the tray to the center of the body. \\
\hline & 8) Pick up a trowel. \\
\hline & 9) Fill $80 \%$ of the 25 -hole tray with the soil with the trowel. \\
\hline & 10) Arrange the top of the tray. \\
\hline & 11) Shake off the soil from your hand. \\
\hline \multirow{5}{*}{ 3. Sowing } & 12) Bring the small dish with seeds to a comfortable place to work. \\
\hline & 13) Place $2-3$ seeds in each cell of the tray. \\
\hline & 14) Cover the seeds with the soil in the bowl with your right hand. \\
\hline & 15) Flatten the surface of the soil. \\
\hline & 16) Shake off the soil from your hand. \\
\hline \multirow{2}{*}{ 4. Writing the label } & 17) Write the name and date of the seeds on the label. \\
\hline & 18) Place the label on the front edge of the tray. \\
\hline \multirow{4}{*}{ 5. Watering } & 19) Grab a watering can with your right hand. \\
\hline & 20) Spray all the water on the tray evenly. \\
\hline & 21) Put down the watering can. \\
\hline & 22) Place your hand down on the table. \\
\hline
\end{tabular}

respectively in order to statistically verify differences in the activity of each muscle between work sections, and differences in each section between the activity of the muscles. The significant level was 0.05 .

\section{Results and Discussion}

This study was conducted among 30 female adults who had an experience of horticultural activities to examine the muscle activity before and after the sowing activity by work section, and the results are as follows.

\section{Work time for each section and muscle activity by muscle type}

Work time for each section and the muscle activity by muscle type were measured and analyzed using descriptive statistics. The average work time for the section of mixing the soil was $76.54 \pm 17.46$ seconds, and the muscle that showed the highest activity was the brachioradialis $(30.91 \pm 20.24 \mu \mathrm{V})$. The average work time for the section of filling the tray with the soil was $180.50 \pm 119.26$ seconds, and the muscle that showed the highest activity was the brachioradialis $(32.50 \pm 16.62 \mu \mathrm{V})$. The average work time for the section of sowing was $229.15 \pm 103.25$ seconds, and the muscle that showed the highest activity was the brachioradialis $(27.80 \pm 15.38 \mu \mathrm{V})$. The average work time for the section of writing 
the label was $31.72 \pm 12.42$ seconds, and the muscle that showed the highest activity was the brachioradialis ( $28.34 \pm 17.88$ $\mu \mathrm{V})$. The average work time for the section of watering was $56.52 \pm 27.39$ seconds, and the muscle that showed the highest activity was the flexor carpi ulnaris $(7.75 \pm 12.75 \mu \mathrm{V})$ (Table 3$)$.

\section{Differences in the activity of each muscle between sections}

Differences in the activity of each muscle between sections were analyzed, and the results are as shown in Fig. 3. The

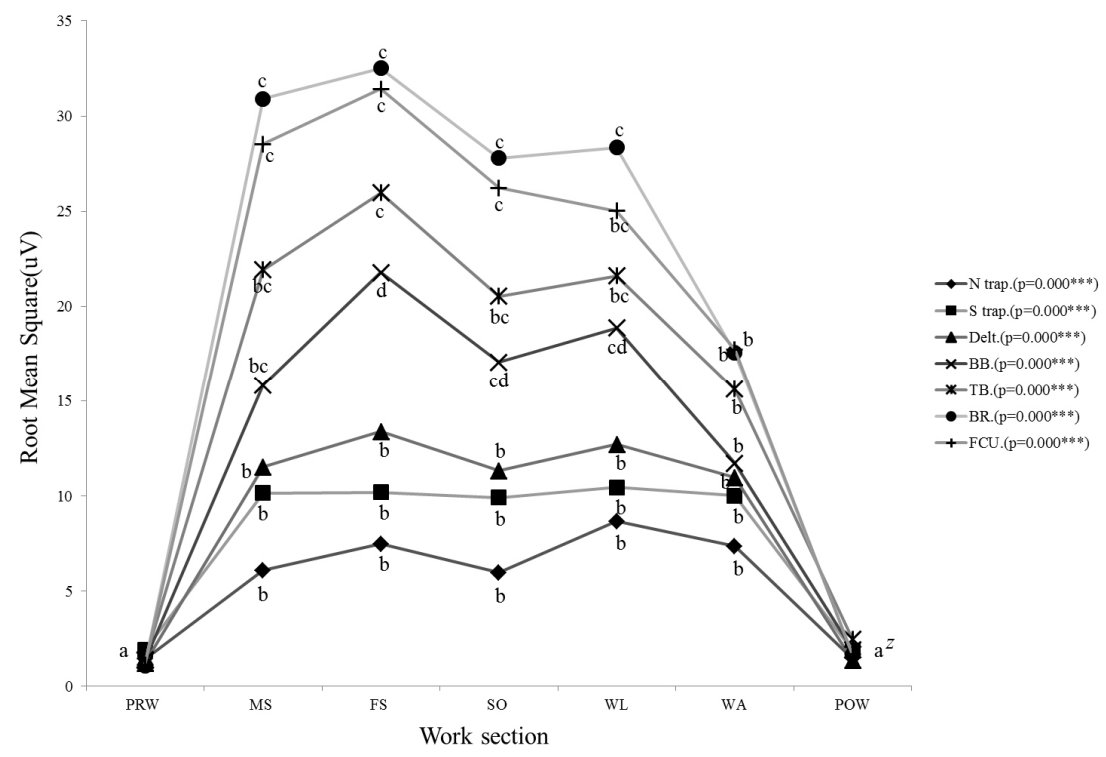

Figure 3. Differences between the muscle activity of muscles in each section.

${ }^{2}$ Different letters are statistically different at $p=.05$ according to Duncan's multiple range test.

PRW=Pre-work; MS=Mixing the soil; FS=Filling the tray with the soil; SO=Sowing; WL=Writing the label; WA=Watering; POW= Post-work; $\mathrm{N}$ trap.=Upper trapezius near the neck; $\mathrm{S}$ trap.=Upper trapezius near the shoulder; Delt.=Middle deltoid; $\mathrm{BB} .=\mathrm{Biceps}$ brachii; TB. $=$ Triceps brachii; BR. $=$ Brachioradialis; FCU. $=$ Flexor carpi ulnaris

*** Significant at $p<.001$ leveled by ANOVA.

Table 3. Muscle activity of each muscle in each work section.

\begin{tabular}{lcccccccc}
\hline & PRW & MS & FS & SO & WL & WA & POW & Total \\
\hline Time $(\mathrm{sec})$ & $60.03 \pm 1.29$ & $76.54 \pm 17.46$ & $180.50 \pm 119.26$ & $229.15 \pm 103.25$ & $31.72 \pm 12.42$ & $56.52 \pm 27.39$ & $60.03 \pm 0.70$ & $694.49 \pm 237.66$ \\
N trap. $(\mu \mathrm{V})$ & $1.43 \pm 1.41$ & $6.08 \pm 4.51$ & $7.47 \pm 6.12$ & $5.96 \pm 4.25$ & $8.66 \pm 7.41$ & $7.36 \pm 6.14$ & $1.47 \pm 1.07$ & $39.38 \pm 26.08$ \\
S trap. $(\mu \mathrm{V})$ & $1.91 \pm 2.07$ & $10.14 \pm 6.70$ & $10.18 \pm 5.83$ & $9.90 \pm 6.58$ & $10.45 \pm 5.84$ & $10.00 \pm 5.16$ & $1.88 \pm 1.97$ & $56.03 \pm 26.99$ \\
Delt. $(\mu \mathrm{V})$ & $1.20 \pm 1.78$ & $11.51 \pm 7.02$ & $13.37 \pm 8.59$ & $11.31 \pm 8.03$ & $12.71 \pm 10.97$ & $10.96 \pm 6.69$ & $1.36 \pm 1.86$ & $63.88 \pm 36.83$ \\
BB $(\mu \mathrm{V})$ & $1.35 \pm 2.31$ & $15.83 \pm 11.43$ & $21.79 \pm 12.32$ & $17.07 \pm 10.72$ & $18.87 \pm 11.53$ & $11.71 \pm 6.37$ & $1.93 \pm 2.22$ & $89.55 \pm 46.28$ \\
TB $(\mu \mathrm{V})$ & $1.39 \pm 1.88$ & $21.92 \pm 17.43$ & $25.96 \pm 15.77$ & $20.53 \pm 15.36$ & $21.61 \pm 16.53$ & $15.62 \pm 10.02$ & $2.48 \pm 2.95$ & $110.53 \pm 64.18$ \\
BR $(\mu \mathrm{V})$ & $1.09 \pm 0.92$ & $30.91 \pm 20.24$ & $32.50 \pm 16.62$ & $27.80 \pm 15.38$ & $28.34 \pm 17.88$ & $17.56 \pm 11.08$ & $1.68 \pm 1.36$ & $142.33 \pm 62.47$ \\
FCU $(\mu \mathrm{V})$ & $1.12 \pm 0.85$ & $28.53 \pm 21.00$ & $31.42 \pm 20.49$ & $26.23 \pm 17.67$ & $25.01 \pm 16.06$ & $17.75 \pm 12.75$ & $1.67 \pm 1.53$ & $134.06 \pm 79.03$ \\
\hline \multicolumn{1}{c}{ Total } & $9.48 \pm 6.30$ & $124.92 \pm 62.63$ & $142.70 \pm 66.06$ & $118.80 \pm 63.50$ & $125.63 \pm 66.09$ & $90.95 \pm 41.54$ & $12.41 \pm 6.92$ & $635.76 \pm 36.83$ \\
\hline
\end{tabular}

Values are Mean \pm standard deviation.

Time $=$ Work time; PRW = Pre-work; MS = Mixing the soil; FS = Filling the tray with the soil; SO = Sowing; WL = Writing the label; WA

= Watering; POW = Post-work; N trap. = Upper trapezius near the neck; $\mathrm{S}$ trap. = Upper trapezius near the shoulder; Delt. = Middle deltoid; BB. = Biceps brachii; TB. $=$ Triceps brachii; BR. = Brachioradialis; FCU. $=$ Flexor carpi ulnaris. 
upper trapezius near the neck, the upper trapezius near the shoulder, and the middle deltoid showed a statistically significant increase in all the 5 sections including mixing the soil, filling the tray with the soil, sowing, writing the label, and watering, compared to the stable state for 1 minute before and after the process of sowing, but there was no statistical difference in the muscle activity between the 5 sections.

The biceps brachii showed a statistically significant increase in all the 5 sections including mixing the soil, filling the tray with the soil, sowing, writing the label, and watering, compared to the stable state for 1 minute before and after the process of sowing, and the muscle activity during the section of watering was highest, followed by mixing the soil, sowing, writing the label, and filling the tray with the soil. In particular, the muscle activity during the section of filling the tray with the soil was statistically significantly higher than the rest 4 sections.

The triceps brachii showed a statistically significant increase in all the 5 sections including mixing the soil, filling the tray with the soil, sowing, writing the label, and watering, compared to the stable state for 1 minute before and after the process of sowing, and the muscle activity during the section of watering was highest, followed by sowing, writing the label, mixing the soil, and filling the tray with the soil. In particular, the muscle activity during the section of filling the tray with the soil was statistically significantly higher than the rest 4 sections.

The brachioradialis showed a statistically significant increase in all the 5 sections including mixing the soil, filling the tray with the soil, sowing, writing the label, and watering, compared to the stable state for 1 minute before and after the process of sowing, and the muscle activity during the sections of mixing the soil, filling the tray with the soil, sowing, and writing the label was statistically significantly higher than the section of watering. There as no statistical difference in the muscle activity between the sections of mixing the soil, filling the tray with the soil, sowing and writing the label.

The flexor carpi ulnaris showed a statistically significant increase in all the 5 sections including mixing the soil,

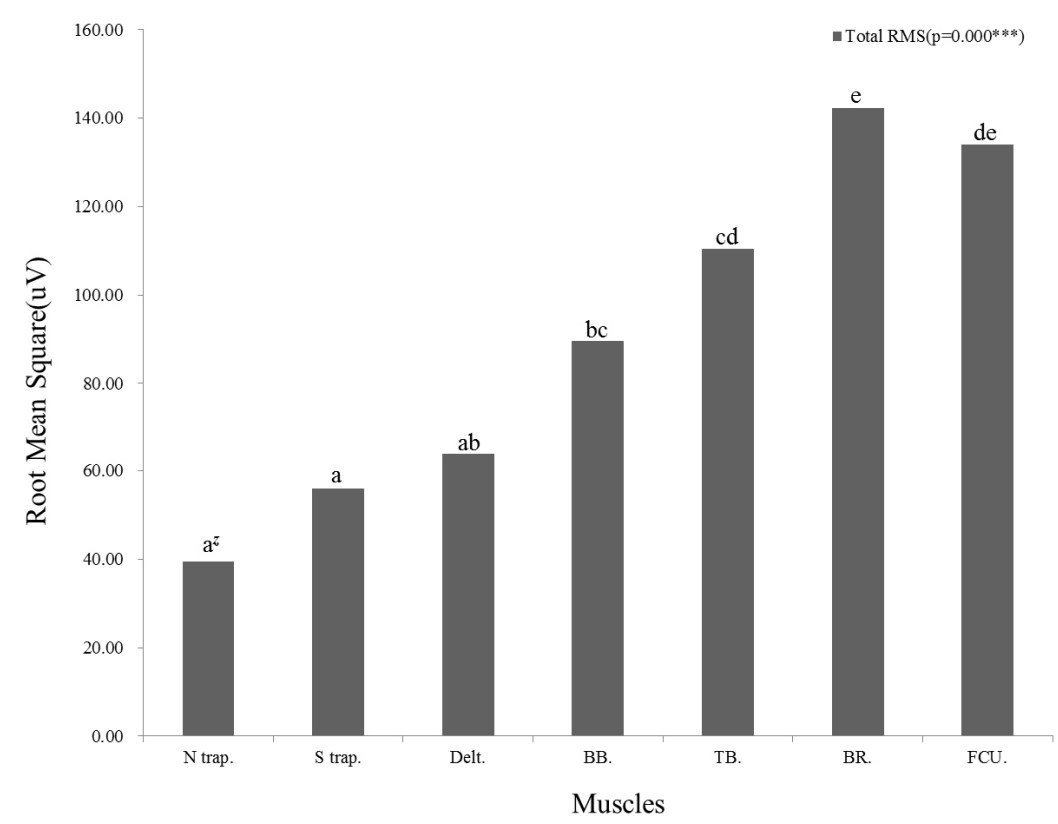

Figure 4. Differences between the muscle activity of muscles.

${ }^{\text {z}}$ Different letters are statistically different at $p=.05$ according to Duncan's multiple range test.

$\mathrm{N}$ trap. $=$ Upper trapezius near the neck; $\mathrm{S}$ trap. $=$ Upper trapezius near the shoulder; Delt. $=$ Middle deltoid; $\mathrm{BB} .=$ Biceps brachii; $\mathrm{TB} .=$ Triceps brachii; BR. $=$ Brachioradialis; FCU. $=$ Flexor carpi ulnaris.

***Significant at $p<.001$ leveled by ANOVA. 
filling the tray with the soil, sowing, writing the label, and watering, compared to the stable state for 1 minute before and after the process of sowing, and the muscle activity during the sections of mixing the soil, filling the tray with the soil, sowing, and writing the label was statistically significantly higher than the section of watering. There as no statistical difference in the muscle activity between the sections of mixing the soil, filling the tray with the soil, sowing and writing the label.

In terms of the activity of each muscle throughout the entire sowing process, there was no statistically significant difference between the upper trapezius near the neck, the upper trapezius near the shoulder, and the middle deltoid, and, compared to the three muscles, the activity of the biceps brachii was highest, followed by the triceps brachii, the flexor carpi ulnaris, and the brachioradialis (Fig. 4).

\section{Differences in each section between the activity of muscles}

Differences in each section between the activity of muscle were analyzed, and the results are as shown in Fig. 5. There was no statistically significant difference between muscle types in the stable state for 1 minute before and after the sowing process.

During the section of mixing the soil, the activity of the upper trapezius near the neck was highest, followed by the upper trapezius near the shoulder, the middle deltoid, the biceps brachii, the triceps brachii, the flexor carpi ulnaris, and the brachioradialis, and, in particular, the activity of the brachioradialis was statistically significantly higher than the rest muscle types.

During the section of filling the tray with the soil, the activity of the biceps brachii, the triceps brachii, the flexor carpi

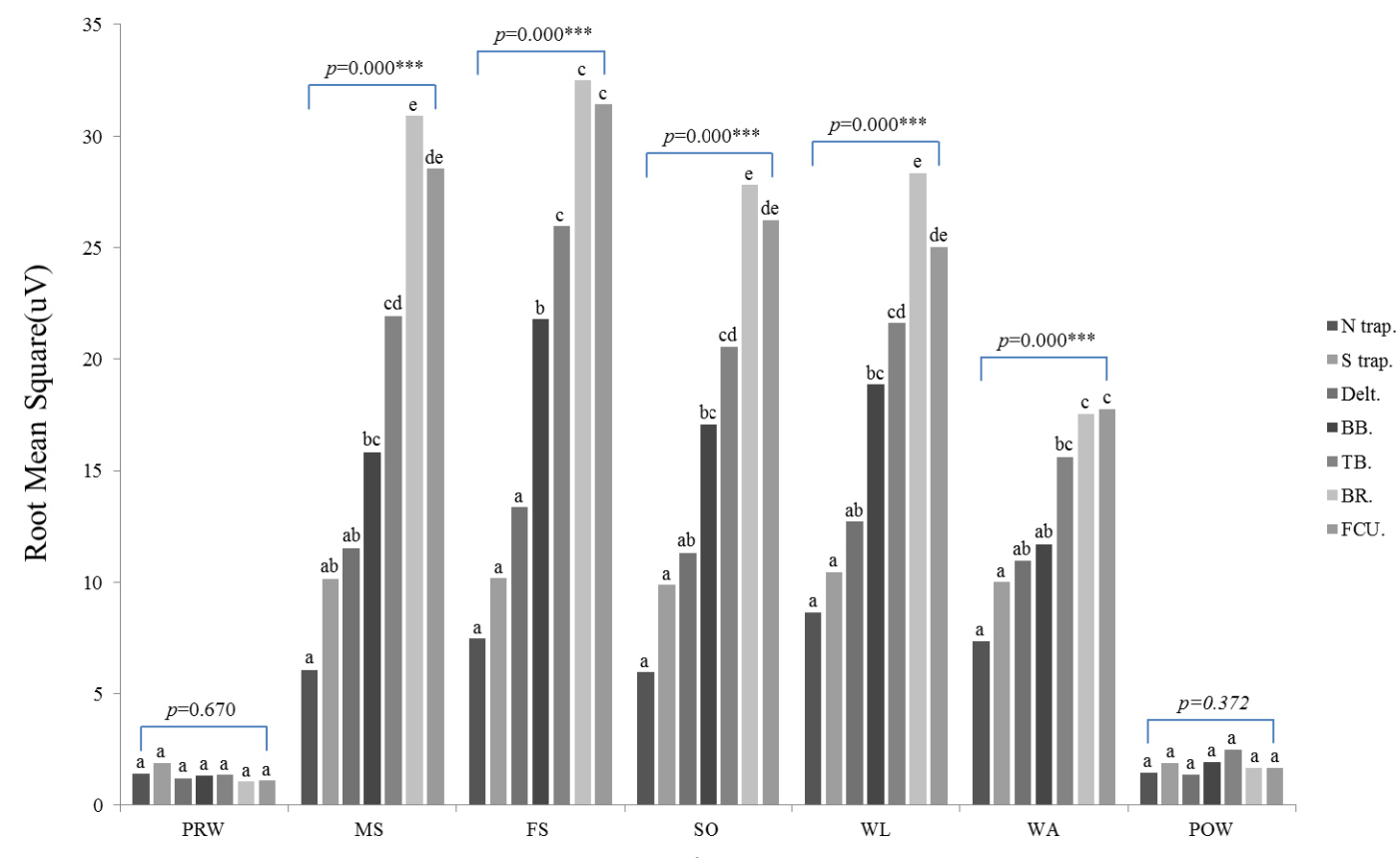

Muscles

Figure 5. Differences between the muscle activity of muscles in each section.

${ }^{\mathrm{z} D i f f e r e n t ~ l e t t e r s ~ a r e ~ s t a t i s t i c a l l y ~ d i f f e r e n t ~ a t ~} p=.05$ according to Duncan's multiple range test.

$\mathrm{PRW}=$ Pre-work; $\mathrm{MS}=$ Mixing the soil; FS $=$ Filling the soil; $\mathrm{SO}=$ Sowing; $\mathrm{WL}=$ Writing the label; $\mathrm{WA}=$ Watering; POW $=$ Post-work; $\mathrm{N}$ trap. $=$ Upper trapezius near the neck; $\mathrm{S}$ trap. $=$ Upper trapezius near the shoulder; Delt. $=$ Middle deltoid; $\mathrm{BB} .=$ Biceps brachii; $\mathrm{TB} .=$ Triceps brachii; BR. $=$ Brachioradialis; FCU. $=$ Flexor carpi ulnaris.

***Significant at $p<.001$ leveled by ANOVA. 
ulnaris, and the brachioradialis, in this order, was statistically significantly higher than that of the upper trapezius near the neck, the upper trapezius near the shoulder, and the middle deltoid.

During the section of sowing, the activity of the middle deltoid, the biceps brachii, and the triceps brachii, in this order, was statistically significantly higher than that of the upper trapezius near the neck, and the upper trapezius near the shoulder, and the activity of the brachioradialis, and the flexor carpi ulnaris was also statistically significantly higher than that of the middle deltoid, the biceps brachii, and the triceps brachii.

During the section of writing the label, the activity of the middle deltoid, the biceps brachii, the triceps brachii, the brachioradialis, and the flexor carpi ulnaris, in this order, was statistically significantly higher than that of the upper trapezius near the neck, and the upper trapezius near the shoulder.

During the section of watering, the activity of the middle deltoid, the biceps brachii, the triceps brachii, the brachioradialis, and the flexor carpi ulnaris, in this order, was also statistically significantly higher than that of the upper trapezius near the neck, and the upper trapezius near the shoulder.

The total muscle activity of the 7 muscles in each work section was compared, and the results showed that the total muscle activity in the 5 sections of mixing the soil, filling the tray with the soil, sowing, writing the label, and watering was statistically significantly higher than that before and after the sowing process. Out of the 5 sections, watering showed the lowest muscle activity, and the muscle activity during the sections of mixing the soil, filling the tray with the soil, sowing and writing the label was statistically significantly higher than the section of watering (Fig. 6).

\section{Discussion}

This study aimed to observe the muscle activity in the right forearm while doing the sowing activity, and to analyze it by muscle type and work section, including before and after doing the sowing activity. All the 7 muscles observed in this

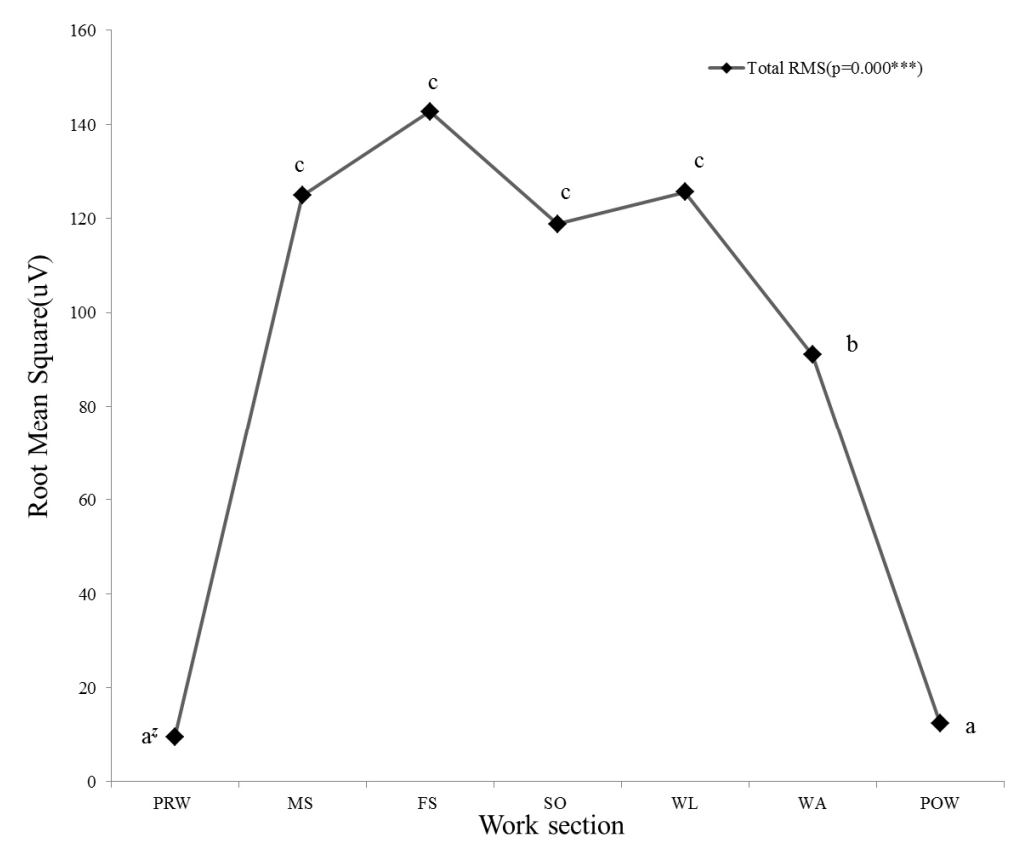

Figure 6. Differences and changes in the total muscle activity in each section.

${ }^{2}$ Different letters are statistically different at $p=.05$ according to Duncan's multiple range test.

PRW = Pre-work; MS = Mixing the soil; FS = Filling the soil; SO = Sowing; WL = Writing the label; WA = Watering; POW = Post-work.

***Significant at $p<.001$ leveled by ANOVA. 
study including the upper trapezius near the neck, the upper trapezius near the shoulder, the middle deltoid, the biceps brachii, the triceps brachii, the brachioradialis, and the flexor carpi ulnaris showed a statistically significant muscle activity in the sowing process. In particular, elbow muscles including the biceps brachii, and the triceps brachii, and forearm muscles including the flexor carpi ulnaris, and the brachioradialis showed a higher muscle activity than shoulder muscles including the upper trapezius near the neck, the upper trapezius near the shoulder, and the middle deltoid, and, in particular, the muscle activity of the brachioradialis was statistically significantly high. These results, however, are not consistent with the results of Lee (2017) that the muscle activity of the shoulder muscles was higher than that of the arm muscles during the sowing activity. In particular, Lee (2017) reported that the muscle activity of shoulder muscles during the sections of reaching seeds, and moving seeds backward and forward was statistically significantly higher than that during the sections of picking up and putting down seeds. However, the results of this study showed that the section of mixing the soil, which involves reaching and moving seeds backward and forward, and also the section of sowing, which involves picking up and putting down seeds showed a significantly high muscle activity in the biceps brachii, the triceps brachii, the flexor carpi ulnaris, and the brachioradialis. In addition, the muscle activity of the biceps brachii and the triceps brachii was high in the section of filling the tray with the soil, which is attributable to the fact that elbows need to be repeatedly moved toward the trunk in order to fill the tray with the soil with a trowel. In particular, the muscle activity of the brachioradialis throughout the sowing process was $142.33 \pm 62.47 \mu \mathrm{V}$. Although it would be difficult to compare it as the absolute value, the value was similar to the muscle activity observed in the downswing motion in a non-athletic group in the study conducted by Kim (2006). To make the downswing motion used in the curve ball in bowling, arms need to be lowered completely to hold the bowling ball side by side next to the right calf, and the right elbow needs to be stretched, not opened sideways, and lowered at a right angle against the shoulder line. The brachioradialis is a muscle that flexes the elbow joint in very active motions such as grasping or picking up objects, and the flexor carpi ulnaris is a muscle that acts to flex and adduct the wrist, and flexes the hand from the wrist joint toward the trunk. The biceps brachii assists with the flexion of the elbow joint, the supination of the forearm, and the flexion of the shoulder joint, and the triceps brachii assists with the extension of the elbow joint, and some shoulder joints (Reese, 2013). In particular, the function of the forearm is essential for activities of daily living such as eating, putting on clothes, and cleaning after urination or defecation (Lee, 2005). One of the aftereffects that stroke patients experience most commonly is forearm dysfunction (Trombly and Radomski, 2002), and the functional restoration of the forearms requires delicate functions such as grasping, and handling (Feys et al., 1998). In this regard, the sowing activity that repeats delicate movements including grasping and putting down seeds can be used as an alternative to activities for the improved function and rehabilitation of the forearms. In addition, according to Lee (2006), flower arrangement requires a combination of several motions such as picking up, reaching, and turning flowers, as well as multiple tasks such as cutting, bending, winding, sticking and rolling. It can also involve tasks in a seated position, and, at the same time, various movements of the forearm. In this study, the sowing process was composed of tasks that require repeated motions such as stretching the hand to reach tools, picking up tools, and moving the forearm back to the center of the body, and motions such as cutting a plastic bag filled with the soil with scissors, picking up seeds and putting them down in the cells of a tray were also repeated, which seemed to induce the movement of the forearm.

\section{Conclusions}

This study was conducted to analyze the muscle activity in the right forearm and shoulder while doing the sowing activity by muscle type and work section, and to examine the possibility of the utilization of sowing as a muscle exercise 
and a rehabilitative activity based on the results of the analysis. It took around 11 minutes to perform the sowing process applied in this study, and during the entire process, all the 7 selected muscles, including the upper trapezius near the neck, the upper trapezius near the shoulder, the middle deltoid, the biceps brachii, the triceps brachii, the brachioradialis, and the flexor carpi ulnaris, showed a statistically significant difference in the muscle activity compared to the stable state. The results indicate that the entire sowing process is effective to activate the upper trapezius near the neck, the upper trapezius near the shoulder, the middle deltoid, the biceps brachii, the triceps brachii, the brachioradialis, and the flexor carpi ulnaris. In particular, the muscle activity of the biceps brachii and the triceps brachii was statistically significantly high in the section of filling the tray with the soil, which indicates that the task of filling the tray with the soil can be utilized as an exercise to activate the biceps brachii and the triceps brachii. In addition, the muscle activity of the brachioradialis was significantly high in all the work sections, which indicates that the sowing process can be utilized to exercise the brachioradialis in particular.

Understanding changes in the muscle activity during the sowing process is a necessary step to identify the muscles that can be exercised through the sowing process, and examine its rehabilitative effects. Therefore, the results obtained in this study can be utilized as the basic data for identifying the types of the muscles that are activated during the sowing process, and their muscle activity levels, and, they can be also used for the overall exercise and rehabilitation of the forearm muscles as well as for the activation of certain muscles.

\section{References}

Feys, H.M., W.J. DeWeerdt, B.E. Selz, G.A. Cox Steck, R. Spichiger, L.E. Vereeck, K.D. Putman, and G.A. Van Hoydonck. 1998. Effect of a therapeutic intervention for the hemiplegic upper limb in the acute phase after stroke : A single-blind, randomized, controlled multi center trial. Stroke 29(4):785-792. DOI: 10.1161/01.STR.29.4.785

Go, H.U., M.G. Kim, S.I. Kim, H.C. Kim, S.H. Park, Y.J. Woo, Y.R. Yoon, H.R. Yoon, G.J. Lee, S.Y. Lee, J.J. Lim, and M.H. Jo. 1997. Digital biomedical signal processing. Yau Moon Gak. Seoul, Korea.

Jang, H.S., K.J. Kim, E.H. Yoo, and H.H. Jung. 2016. Visual preference and emotional change of user on the shape of cut-flower at public indoor space. J. Korean Soc. People Plants Environ. 19(5):427-434. DOI: 10.11628/ksppe.2016.19.5.427

Kim, T.H. 2006. Analysis on electromyogram according to the ball throwing motion of bowling players. MS Thesis, Kyungnam Univ., Changwon, Korea.

Lee, A.Y. 2017. Analysis of kinematic and kinetic characteristics of horticultural therapy activity and verification of its rehabilitative effectiveness. PhD. Diss., Konkuk Univ., Seoul, Korea.

Lee, S.M., A. Kaufman, and J.K. Suh. 2012. Analysis of pulse on three sensory stimulations of three herbal plants. J. Korean Soc. People Plants Environ. 15(4):241-247.

Lee, S.S. 2006. Approach to rehabilitative horticultural therapy based on motion analysis of flower arrangement. PhD. Diss., Konkuk Univ., Seoul, Korea.

Lee, S.S., S.A. Park, O.Y. Kwon, J.E Song, and K.C. Son. 2012. Measuring range of motion and muscle activation of flower arrangement tasks and application for improving upper limb function. Korean J. Hort. Sci. Technol. 30(4):449-462.

Lee, T.Y. 2005. Daily activities. Topmediopia, Seoul, Korea.

Lim, L.R. 2016. Analysis of horticultural therapy programs of korean master's and doctorate degree dissertations for general adult. MS Thesis, Korea Univ., Seoul, Korea.

Matsuo, E. 1998. Up-to-date trend of horticulture therapy in Japan. J. Korean Soc. People Plants Environ. 1(1):22-33.

Netter, F.H. 2000. The ciba collection of medical illustrations: Volum 8 part I musculoskeletal system anatomy, physiology, and metabolic disorders. The compilation committee of CIBA color medical illustrations eds. Jung Dam Press, Seoul, Korea. 
Park. S.A., A.Y. Lee, H.S. Lee, K.S. Lee, and K.C. Son. 2015. A comparison of exercise intensity between two horticultural and four common physical activities among male adults in their 20s. Kor. J. Hort. Sci. Technol. 33(1):133-142. DOI: 10.7235/hort.2015.14083

Reese, N.B. 2013. Muscle and sensory testing 3th. ed. Jang S.K. et al. eds. Youngmoon press, Seoul, Korea.

Relf, D. 2006. Volunteers: Implementation therapeutic horticulture or supporting horticultural therapy, p 23-44. Korean Horticultural Therapy Association. Seoul, Korea.

Trombly, C.A. and M.V. Radomski. 2002. Occupational therapy for physical dysfunction. $5^{\text {th }}$ ed. Lippincott Williams \& Wilkins, Baltimore, USA. 